\title{
Analysis of Relationship between Burnout and Emotional Intelligence of Special School Teachers
}

\author{
Ting Deng ${ }^{1}$, Danna $\mathrm{Luo}^{2}$, Ma Jinjing ${ }^{3}$, Xie $\mathrm{Ma}^{*}$ \\ 1,2,3,* Department of Education, Yunnan Normal University Key Laboratory of EducationalInformation for \\ Nationalities, Yunnan Normal University \\ *Corresponding author. Email: mxnn6@aliyun.com
}

\begin{abstract}
This study used the MBI-GS and EIS to investigate the status of burnout and its relationship with the emotional intelligence of 146 special school teachers. The results showed that : (1) the burnout score of special school teachers was slightly higher than the theoretical median, and their burnout level was above the average, which was $60.27 \%$. (2) There are significant differences in the total score of burnout and the emotional intelligence level of each dimension. The higher the emotional intelligence level is the less likely to show burnout related symptoms. (3) The dimensions of emotional intelligence, such as emotion perception, regulating the emotions of others, and emotion application, are negatively correlated with the dimensions of burnout. (4) Regression analysis showed that emotion perception could negatively predict emotional exhaustion and low personal accomplishment, whereas regulating the emotions of others could negatively predict low personal accomplishment. Emotional intelligence of special school teachers can be used as one of the predictors of burnout. Emotional intelligence training can effectively alleviate burnout of special school teachers.
\end{abstract}

Keywords: special schools, teachers, burnout, emotional intelligence, relationships.

\section{INTRODUCTION}

Teachers' burnout refers to a kind of overreaction when teachers are unable to cope with pressure well at work, and a state of exhaustion in emotion, attitude, and behavior under long-term excessive pressure [1]. According to some researchers, special school teachers are prone to burnout because of the specificity of their work environment. For example, M. Wang et al. found that the level of job burnout of special school teachers was above the average, and the score of emotional exhaustion was as high as 4.17 , much higher than the median level [2]. L. F. Wang also found in his study that $17.6 \%$ of special school teachers showed obvious emotional exhaustion, and $12.8 \%$ of special school teachers showed low personal accomplishment [3]. The long-term maintenance of a high level of burnout by special school teachers will have a negative impact on teachers' personal development, life status, students' mental health, academic achievement, and other aspects. Therefore, more and more researchers have paid attention to the current situation of burnout of special school teachers and its influencing factors, such as teachers' emotional intelligence [4]-[7].
The emotional intelligence of teachers refers to the sum of the objective abilities of individual teachers to accurately identify and use the monitored emotional information to guide educational thoughts and improve teaching behaviors to maintain the smooth progress of teaching activities [8]. Emotional intelligence can regulate work-family conflict, and those with higher emotional intelligence are able to manage the role conflict between the two and have more positive psychological resources to devote to work and family [9]. This is due to the fact that teachers with a high level of emotional intelligence are able to regulate their bad emotions at work and allow themselves to carry out teaching activities in a positive state, thus improving work efficiency and job satisfaction. Otherwise, they are prone to symptoms related to burnout such as emotional exhaustion, low personal accomplishment, and low job engagement [10].

The relationship between burnout and emotional intelligence has attracted the attention of many researchers. Most studies have found a significant negative correlation between burnout and emotional intelligence, but the conclusions of the relationship are inconsistent in individual dimensions. J. H. Yao et al. 
found that teachers' emotional regulation ability and emotional perception ability were significantly negatively correlated with emotional exhaustion, cognitive exhaustion, and low personal accomplishment in job burnout, and teachers' emotional understanding ability also significantly predicted the degree of depersonalization [7]. F. Tang also concluded that teachers' burnout could be effectively resolved by improving their emotional intelligence [8]. However, most of the groups studied in the relationship between the two are mainly general school teachers, and there is a lack of research studies on burnout and emotional intelligence of special school teachers at home and abroad. Therefore, this study takes special school teachers as the research object, explores the current situation of their burnout and the differences of their burnout performance under different levels of emotional intelligence, and analyzes the relationship between burnout and emotional intelligence of special school teachers to provide ideas for the healthy development of special school teachers' career.

\section{METHODS AND SUBJECTS}

\subsection{Subjects}

In this study, 166 special school teachers were surveyed by random sampling. After eliminating the questionnaires with incomplete information and wrong information, 146 valid questionnaires remained, and the effective recovery rate was $87.95 \%$. Among them, 35 (23.97\%) were male teachers and 111 (76.03\%) were female teachers, their ages ranged from 20 to 58 years old, with an average age of 36.97 years; and their average teaching experience was 14.50 years.

\subsection{Research instruments}

Burnout Scale. In this study, Maslach Burnout Inventory General Surve (MBI - GS) was used, which was divided into 3 dimensions of emotional exhaustion, depersonalization, and low personal fulfillment, with a total of 16 questions. Emotional exhaustion included 5 questions, and positive score was adopted. The depersonalization consisted of 5 questions, which were scored positively. A total of 6 questions with low personal accomplishment were scored in reverse. The Liker 5-point scale was used, with a scale of 1 to 5 from non-conformity to conformity, with higher scores representing higher levels of burnout. The Cronbach's a indicate of the scale was 0.715 , and the Cronbach's a indicate of each factor was $0.919,0.768$, and 0.846 , respectively, which can be used as a valid assessment tool.

Emotional Intelligence Scale. In this study, the Emotional Intelligence Scale (EIS) developed by Schutte et al. was used, which consists of 4 dimensions: emotion perception, regulating the emotions of others, regulating oneself's emotions, and emotion application, with a total of 33 questions. There were 12 questions for emotion perception, 10 questions for regulating the emotions of others, 6 questions for regulating oneself's emotions, and 5 questions for emotion application, all of which were scored positively. Liker5-point scoring method was adopted, with 1 point representing inconformant and 5 points representing conformant. The higher the score, the higher the emotional intelligence. The Cronbach's a of the scale is 0.724 , which has good reliability and validity.

\subsection{Data processing}

SPSS22.0 was used for statistical and analysis of the data.

\section{RESULTS}

\subsection{Overall characteristics of special school teachers' burnouts}

The results show that the total score of burnout of special school teachers is $(3.16 \pm 0.92)$, indicating that this group has a certain degree of burnout. From the dimensions of burnout, emotional exhaustion was (2.48 \pm $0.94)$ points, depersonalization was $(2.10 \pm 0.78)$ points, and low personal accomplishment was $(1.99 \pm 0.69)$ points. It can be seen that the score of emotional exhaustion of special school teachers is close to the median level, and the phenomenon of emotional exhaustion is more serious. The scores of depersonalization and low personal accomplishment were both lower than the theoretical median (3 points), indicating that special school teachers have a warm and positive attitude towards students and work. Based on the theoretical median, the study found that the burnout rate of special school teachers was $60.27 \%$, the emotional exhaustion rate and the depersonalization rate were $31.51 \%$ and $17.12 \%$, respectively, and the proportion of low personal accomplishment was $11.64 \%$. See Table 1.

Table1. Overall characteristics of special school teachers' burnouts

\begin{tabular}{|c|c|c|c|c|}
\hline & Emotional exhaustion & Depersonalization & Low personal accomplishment & Total Burnout Score \\
\hline$M \pm S D$ & $2.48 \pm 0.94$ & $2.10 \pm 0.78$ & $1.99 \pm 0.69$ & $3.16 \pm 0.92$ \\
\hline$\%$ & $31.51 \%$ & $17.12 \%$ & $11.64 \%$ & $60.27 \%$ \\
\hline
\end{tabular}




\subsection{Differential analysis of burnout among special school teachers with different levels of emotional intelligence}

Referencing the classification standard of teachers' emotional intelligence by J. H. Yao et al. , the top 27\% and bottom $27 \%$ of the total average score of emotional intelligence were taken as the dividing point [7]. In this study, teachers' emotional intelligence scores below 3.52 were classified as low, above 4.36 as high, and between 3.52 and 4.36 as moderate. According to this standard, the emotional intelligence level of special school teachers is divided into: high level group, medium level group, and low level group.

The results showed that there was statistical significance in the emotional intelligence level of special school teachers' burnout. The total score of burnout, emotional exhaustion, depersonalization, and low personal accomplishment were all decreased with the increase of emotional intelligence ( $F=20.368,10.591$, $6.578,21.619, \mathrm{P}<0.01)$. The post-mortem test found that in the total score of burnout and the dimension of low personal accomplishment, the high level emotional intelligence group was significantly lower than the medium level group, and the low level and medium level emotional intelligence group were significantly higher than the high level group $(\mathrm{P}<0.05)$. In terms of emotional exhaustion and depersonalization, the high emotional intelligence group was significantly lower than the medium and low emotional intelligence groups $(\mathrm{P}<0.05)$, but there was no significant difference between the other groups. See table 2 .

Table2. Differential analysis of burnout among special school teachers with different levels of emotional intelligence

\begin{tabular}{|c|c|c|c|c|}
\hline & $\begin{array}{c}\text { Emotion } \\
\text { perception }\end{array}$ & Regulating the emotions of others & $\begin{array}{c}\text { Regulating oneself's } \\
\text { emotions }\end{array}$ & $\begin{array}{c}\text { Emotion } \\
\text { application }\end{array}$ \\
\hline $\begin{array}{c}\text { Emotional } \\
\text { exhaustion }\end{array}$ & $-0.447^{\star \star}$ & $-0.292^{\star \star}$ & -0.09 & $-0.244^{\star \star}$ \\
\hline Depersonalization & $-0.337^{\star \star}$ & $-0.237^{\star \star}$ & -0.052 & $-0.229^{\star \star}$ \\
\hline $\begin{array}{c}\text { Low personal } \\
\text { accomplishment }\end{array}$ & $-0.520^{\star \star}$ & $-0.446^{\star \star}$ & -0.136 & $-0.265^{\star \star}$ \\
\hline
\end{tabular}

Note: ${ }^{* *} p<0.01, * * * p<0.001$

\subsection{Correlation analysis between burnout and emotional intelligence of special school teachers}

In order to explore the relationship between the dimensions of burnout, emotional exhaustion, depersonalization, low personal accomplishment, and the dimensions of emotional intelligence, emotion perception, regulating the emotions of others, regulating oneself's emotions, and emotion application of special school teachers, the correlation analysis was carried out. The results showed that there was a significant negative correlation between the other dimensions of emotional intelligence and the dimensions of burnout, except that there was no significant correlation between regulating oneself's emotions and emotional exhaustion, depersonalization and low personal accomplishment. Teachers' emotion perception were negatively correlated with emotional exhaustion, depersonalization, and low personal accomplishment ( $r=-0.447,-0.337,-0.520$, all $P<0.01)$. Regulating the emotions of others was negatively correlated with emotional exhaustion, depersonalization, and low personal accomplishment $(r=-0.292, \quad-0.237, \quad-0.446$, all $P<0.01)$. Emotional application was negatively correlated with emotional exhaustion, depersonalization, and low personal accomplishment $(r=-0.244,-0.229,-0.265, P<0.01)$. See table 3.

Table3. The overall level of burnout among special school teachers

\begin{tabular}{|c|c|c|c|c|c|}
\hline Dependent variable & Independent variable & $\beta$ & $t$ & $R^{2}$ & $F$ \\
\hline Emotional exhaustion & Emotion perception & -0.443 & $-4.190^{\star \star \star}$ & 0.210 & $9.394^{\star \star \star}$ \\
\hline & Regulating the emotions of others & 0.009 & 0.076 & & \\
\hline & Regulating oneself's emotions & 0.077 & 0.825 & & \\
\hline & Emotion application & -0.069 & -0.821 & & \\
\hline Depersonalization & Emotion perception & -0.298 & -2.686 & 0.129 & $5.226^{\star *}$ \\
\hline & Regulating the emotions of others & -0.045 & -01.369 & & \\
\hline & Regulating oneself's emotions & 0.081 & 0.831 & & \\
\hline & Emotion application & -0.097 & -1.097 & & \\
\hline Low personal & Emotion perception & -3.393 & $-3.961^{\star \star *}$ & 0.303 & \multirow{2}{*}{$15.310^{\star \star \star}$} \\
\hline & Regulating the emotions of others & -0.248 & $-2.250^{\star}$ & & \\
\hline & Regulating oneself's emotions & 0.146 & 1.668 & & \\
\hline
\end{tabular}

Note: $* * p<0.01, * p<0.001$ 


\subsection{Correlation analysis between burnout and emotional intelligence of special school teachers}

In order to test whether there is a predictive effect of emotional intelligence of special school teachers on their burnout and to examine the magnitude of the predictive power, the linear regression analysis was carried out using emotion perception,regulating the emotions of others, regulating oneself's emotions, and emotion application as the predictive variables, and emotional exhaustion, depersonalization, and low personal accomplishment as the dependent variables. The results showed that emotional perception entering the regression equation negatively predicted emotional exhaustion and low personal accomplishment $(\beta=3.961$, -4.190, $P<0.001$ ). Regulating the emotions of others into the regression equation could negatively predict low personal accomplishment $(\beta=2.250, P<0.05)$. See table 4.

Table 4: The overall level of burnout among special school teachers

\begin{tabular}{|c|c|c|c|c|}
\hline & \multicolumn{3}{|c|}{ Emotional intelligence } & \multirow{2}{*}{$F$} \\
\hline & low level & $\overline{\text { medium level }}$ & high level & \\
\hline Emotional exhaustion & $2.12 \pm 0.81$ & $2.40 \pm 0.86$ & $2.99 \pm 0.99$ & $10.591^{* * *}$ \\
\hline Depersonalization & $1.84 \pm 0.60$ & $2.07 \pm 0.77$ & $2.43 \pm 0.86$ & $6.578^{\star \star}$ \\
\hline Low personal accomplishment & $1.58 \pm 0.57$ & $1.97 \pm 0.61$ & $2.45 \pm 0.63$ & $21.619 * \star \star$ \\
\hline Total Burnout Score & $2.66 \pm 0.77$ & $3.10 \pm 0.83$ & $3.80 \pm 0.86$ & $20.368^{\star \star \star}$ \\
\hline
\end{tabular}

Note: ${ }^{*} p<0.05$, ** $p<0.01$.

\section{DISCUSSION}

\subsection{Analysis of burnout characteristics of special school teachers}

This study found that overall special school teachers' burnout was at a moderate to high level, which is consistent with the research conclusion of Y. Z. Li et al. [11]. In this study, it was found that $60.27 \%$ of teachers in special schools showed symptoms related to burnout, such as powerlessness and confusion about their work and indifference to their teaching subjects. However, Z.H.Liu et al. [12] drew different conclusions in their studies, finding that the level of burnout of teachers in special schools was low and lower than that of teachers in general schools. This may be related to the geographical differences of the subjects, as by Z. H. Liu et al.'s study was based on special school teachers in developed regions such as Beijing, Shenzhen, and Shanghai, whereas Y. Z. Li et al.'s study and the present study were based on special school teachers in less developed regions such as Sichuan, Yunnan, and Henan. Based on the data from X. D. Zeng et al.'s study, it was found that teachers' salary levels in less developed regions would generally be lower than those in developed regions [13]. Salary levels are closely related to economic pressure and personal fulfillment, so the burnout levels of special school teachers in less developed regions are relatively more severe.

\subsection{Analysis of the relationship between burnout and emotional intelligence of special school teachers}

This study found that different emotional intelligence levels of special school teachers have significant differences in the overall level of burnout, emotional exhaustion, depersonalization, and low personal accomplishment. The higher the level of emotional intelligence, the less likely it is to show burnout, which is consistent with the research conclusions of J. H. Yao et al. [7]. Through the correlation analysis, it was found that emotion perception, regulating the emotions of others, and emotion application were significantly negatively correlated with emotional exhaustion, depersonalization, and low personal accomplishment, except that there was no significant correlation between regulating oneself's emotions and emotional exhaustion, depersonalization, and low personal accomplishment. It shows that the higher the emotional intelligence level of special school teachers, the less likely they are to suffer from burnout, emotional exhaustion, depersonalization, and other undesirable phenomena, and the more able they are to deal with the teaching work in a positive psychological state, and experience high personal accomplishment and job satisfaction [14]. On this basis, the regression analysis of the relationship between the two found that special school teachers' ability to emotion perception, regulating the emotions of others negatively predicted their low personal accomplishment, and their ability to emotion perception negatively predicted emotional exhaustion. This suggests that special school teachers' emotional intelligence can predict burnout to some extent, which is consistent with the findings of Reza et al [15]. Therefore, in order to reduce the level of burnout of special school teachers, the emotional intelligence level can be regarded as one of the key factors, especially the ability to emotion perception and regulating the emotions of others.

\section{Conclusion}

Through the above research and analysis, this research has drawn the following conclusions: (1) The level of burnout of special school teachers is above the average. The proportion of special school teachers with obvious burnout is $60.27 \%$, and the proportion of special school teachers showing obvious emotional 
exhaustion, depersonalization, and low personal accomplishment is $31.51 \%, 17.12 \%$, and $11.64 \%$ respectively. (2) Burnout, emotional exhaustion, depersonalization, and low personal accomplishment scores of special school teachers were significantly different in emotional intelligence, the high level group was significantly lower than the middle level group and the low level group, and the middle level group was significantly lower than the low level group. (3) There is a significant negative correlation between burnout and emotional intelligence of special school teachers, and emotional intelligence has a negative predictive effect on burnout.

\section{AUTHORS' CONTRIBUTIONS}

This research was completed with the joint efforts of three authors: X. Ma, Y Tao and T. Deng conducted the research; and T. Deng analyzed the data; T. Deng and D.N. Luo wrote the paper; all authors had approved the final version.

\section{ACKNOWLEDGMENTS}

We are grateful to all the participants for their contributions to the data collection, as well as Ms. Liu for revising the content, grammar, and format of the article.This study was supported by natural science foundation "cognitive control of ethnic bilinguals" (31660282) and the Yunnan Provincial Philosophy and Social Science Foundation "Innovative mechanism of minority middle school students' English syntax learning"(QN2017043).

\section{REFERENCES}

[1] T. J. Kalliath, M. P. O"Driscoll, and D. F. Gillespie, A test of the Maslach Burnout Inventory in three samples of health professionals. Work and Stress, 2020, Vol. 14(1), pp. 41. DOI: https://doi.org/10.1080/026783700417212

[2] M. Wang, C. M. Zhang, On the Relationship Between Special Education Teachers Psychological Capital and Job Burnout. Chinese Journal of Special Education, 2015, Vol. (09), pp. 49-57. DOI: https://doi.org/10.3969/j.issn.1007-3728.2015.09.0 08

[3] L. f. Wang, Job Stress, Coping Strategies, and Job Burnout of Special Education Teachers. Chinese Journal of Special Education, 2010, Vol. (01), pp. 55-59. DOI:

https://doi.org/10.3969/j.issn.1007-3728.2010.01.0 11

[4] N. Petkovic, D. P. Macesic, and V. Balos, Burnout Syndrome among Special Education Professionals.
Healthmed, 2015, Vol. 6(10), pp. 3403-3412. DOI https://doi.org/10.1002/14651858.CD005367.pub3

[5] Y. X. Li, Social Factors Analysis of Professional Burnout of Teachers in Special Schools. Educational Research and Experimentation, 2013, Vol. (04), pp. 71-74. DOI: https://doi.org/CNKI:SUN:YJSY.0.2013-04-016

[6] J. Williams, C. Dikes, The Implications of Demographic Variables as Related to Burnout among a Sample of Special Education Teachers. Education, 2015, Vol. 135(03), pp. 337-345(9).

[7] J. H. Tao, H. J. Guan, A Study of the Relationship between Elementary and Secondary School Teachers' Emotional Intelligence and Job Burnout. Jourmal Of Educational Studies, 2013, Vol. 9(03), pp. 100-110. DOI: https://doi.org/10.3969/j.issn.1673-1298.2013.03.0 13

[8] F. Tang, The Relationship between Emotional Intelligence and Job Burnout of Secondary Vocational School Teachers. Education and Career, 2020. Vol. 24, pp. 90-94. DOI: https://doi.org/10.13615/j.cnki.1004-3985.2020.24. 019

[9] Z. Y. Li, Emotional Intelligence as a Moderator in the Mental Mechanism of Emotional Exhaustion among Preschool Teachers: From the Perspective of Work -Family Conflict. Studies of Psychology and Behavior, 2016, Vol. 14(04), pp. 492-500.

[10] M. Hou, Q. Jiang, C. Xiao, et al., Psychological development and education, 2014, Vol. 30(02), pp. 160-168. DOI:

https://doi.org/10.16187/j.cnki.issn1001-4918.2014 .02 .012

[11] Y. Z. Li, Occupational Stress and Job Burnout among Special Education Teachers: the Moderating Effect of Psychological Capital. Chinese Journal of Special Education, 2014, Vol. (06), pp. 78-82. DOI: https://doi.org/10.3969/j.issn.1007-3728.2014.06.0 13

[12] Z. H. Liu, Analysis on The Characteristics of Teachers' Job Burnout in Special Schools. Chinese Journal of Special Education, 2006, Vol. (04), pp. 71-75. DOI: https://doi.org/10.3969/j.issn.1007-3728.2006.04.0 15

[13] X. D. Zeng, W. J. Yi, The Issues Related to Legitimizing Regional Difference of Teachers' Salary in China. Journal of Central China Normal University (Humanities and Social Sciences), 2015, Vol. 54(05), pp. 155-161. 
[14] M. Platsidou, Trait Emotional Intelligence of Greek Special Education Teachers in Relation to Burnout and Job Satisfaction. School Psychology International, 2010, Vol. 31(1), pp. 60-76. DOI: https://doi.org/10.1177/0143034309360436
[15] P. Reza, S. Samaneh, Personality and Emotional Intelligence in Teacher Burnoutm. Spanish Journal of Psychology, 2012, Vol. 15(1), pp. 227-236. DOI: https://doi.org/10.5209/rev_SJOP.2012.v15.n1.373 14 